\title{
Association of stress hyperglycemia ratio with intracoronary thrombus burden in diabetic patients with ST-segment elevation myocardial infarction
}

\author{
Jiapeng Chu\#, Jiani Tang", Yan Lai, Yanhua Gao, Zi Ye, Chunyu Guan, Keke Ding, Yian Yao, Fei Chen, \\ Xuebo Liu \\ Department of Cardiology, Tongji Hospital, Tongji University, Shanghai, China \\ Contributions: (I) Conception and design: X Liu; (II) Administrative support: X Liu; (III) Provision of study materials or patients: J Chu, J Tang; (IV) \\ Collection and assembly of data: J Chu, Y Lai, Y Gao, Z Ye, C Guan, K Ding, Y Yao; (V) Data analysis and interpretation: J Chu, J Tang, F Chen; (VI) \\ Manuscript writing: All authors; (VII) Final approval of manuscript: All authors. \\ \#These authors contributed equally to this work. \\ Correspondence to: Xuebo Liu, MD. No.389, Xincun Rd, Putuo District, Shanghai 200065, China. Email: liuxuebo70@126.com.
}

Background: Large intracoronary thrombus burden is not rare during primary percutaneous coronary intervention (PCI) in patients with acute ST-segment elevation myocardial infarction (STEMI). Stress hyperglycemia is independently associated with poor prognosis. However, the underlying relationship between stress hyperglycemia and thrombus burden remains unknown. This study aims to investigate the association of stress hyperglycemia, evaluated by the combination of acute and chronic glycemic levels, with intracoronary thrombus burden in diabetic patients with STEMI.

Methods: We enrolled 227 consecutive diabetic patients with STEMI undergoing primary PCI within 12 hours after symptom onset. Stress hyperglycemia was estimated using the stress hyperglycemia ratio (SHR), which was calculated as admission glycemia divided by estimated average glucose derived from glycosylated hemoglobin. Based on reclassified angiographic thrombolysis in myocardial infarction (TIMI) thrombus grades, patients were divided into small thrombus burden (STB) group (TIMI thrombus grades <4) and large thrombus burden (LTB) group (TIMI thrombus grades 4 or 5 ).

Results: Of the entire study population, 77 (33.9\%) patients were categorized as LTB group, whereas 150 (66.1\%) patients presented with STB. The mean age was 64.1 years, and $80.6 \%$ of the patients were male. The SHR levels were significantly higher in patients with LTB than in those with STB [1.31; interquartile range (IQR): 1.13-1.48 versus 1.11; IQR: 0.96-1.32; $\mathrm{P}<0.001]$. The predictive performance of SHR for LTB was moderate (area under the curve: 0.669; 95\% confidence interval: 0.604-0.730; $\mathrm{P}<0.001$ ), with the best cut-off value 1.19 (sensitivity 71.4\%, specificity 64.7\%). The incidence of LTB with SHR $\geq 1.19$ was significantly higher compared with SHR $<1.19$ (50.9\% versus $18.5 \% ; \mathrm{P}<0.001$ ). Based on the multivariable logistic regression analysis, the high SHR $(\geq 1.19)$ was found to be an independent predictor of LTB following adjustment for baseline clinical confounders.

Conclusions: A high SHR value was independently associated with large thrombus burden and has a better predictive value than glycemia at admission in diabetic patients with STEMI undergoing primary PCI. Stress hyperglycemia may play an important role on the intracoronary thrombus formation.

Keywords: Acute hyperglycemia; diabetes mellitus; ST-segment elevation myocardial infarction (STEMI); intracoronary thrombus

Submitted Jun 02, 2020. Accepted for publication Sep 23, 2020.

doi: $10.21037 /$ jtd-20-2111

View this article at: http://dx.doi.org/10.21037/jtd-20-2111

(c) Journal of Thoracic Disease. All rights reserved. 


\section{Introduction}

In patients with ST-segment elevation myocardial infarction (STEMI), large intracoronary thrombus burden is an independent predictor of adverse cardiac outcomes and is associated with increased risk of unsuccessful angiographic reperfusion and distal embolization during primary percutaneous coronary intervention (PCI) (1-3). The early recognition and appropriate treatment of thrombus burden may contribute to the management of STEMI. Admission hyperglycemia is common in patients with acute myocardial infarction (AMI) and independently associated with poor prognosis, particularly in patients without diabetes mellitus (DM) (4,5). This emphasizes the importance of an acute glycemic rise by stress hyperglycemia rather than the levels of glucose at hospital admission.

The admission glycemia has been used as an index of stress hyperglycemia in clinical practice. However, it cannot reflect the "true" acute glycemic increase in diabetic patients with chronic elevation of glucose levels. Recently, the stress hyperglycemia ratio (SHR), which is calculated by combining acute (admission glucose level) and chronic [estimated by glycosylated hemoglobin (HbA1c)] glycemic value, was proposed as a novel index of stress hyperglycemia and had a better prognostic value in AMI patients with DM (6-8). Besides, a recent study has reported that admission hyperglycemia is associated with large thrombus burden (LTB) in nondiabetic patients with STEMI (9). However, the association between stress hyperglycemia and intracoronary thrombus burden in diabetic patients has not been evaluated.

In this study, we aimed to investigate the possible association of SHR, as compare with admission glycemia, with intracoronary thrombus burden in diabetic patients with STMEI treated with primary PCI.

We present the following article in accordance with the STROBE reporting checklist (available at http://dx.doi. org/10.21037/jtd-20-2111).

\section{Methods}

\section{Study populations}

This was a retrospective analysis of consecutive STEMI patients prospectively enrolled who had undergone primary PCI at Tongji Hospital, Tongji University, Shanghai $\leq 12$ hours from onset of symptoms. The inclusion criteria were STEMI on admission with a diagnose of DM. Exclusion criteria included time from symptom onset to balloon
$>12$ hours, severe renal diseases, active infection, cardiogenic shock, previous coronary artery bypass graft surgery and those who underwent planned coronary artery bypass graft. This study was conducted in accordance with the Declaration of Helsinki (as revised in 2013). The study was approved by ethics board of Tongji Hospital of Tongji University (No. KYSB-2014-054) and informed consent was taken from all the patients. STEMI and DM were defined based on the criteria by the European Society of Cardiology and the American Diabetes Association. STEMI was diagnosed if a patient had continuous chest pain associated with elevation of troponin I level lasting $>30$ minutes, and new ST-elevation at the J-point in two contiguous leads with cut-off points: $\geq 2 \mathrm{~mm}$ in men $(\geq 2.5 \mathrm{~mm}$ in men $<40$ years) or $\geq 1.5 \mathrm{~mm}$ in women in leads $\mathrm{V} 2-\mathrm{V} 3$ and/or $1 \mathrm{~mm}$ in other leads, or as the presence of newly developed left bundle branch block (10). The diagnosis of $\mathrm{DM}$ is based on HbA1c or fasting plasma glucose, and on oral glucose tolerance test if still in doubt. DM was defined as $\mathrm{HbA1c} \geq 6.5 \%(\geq 48 \mathrm{mmol} / \mathrm{mol})$, fasting plasma glucose $\geq 126 \mathrm{mg} / \mathrm{dL}(\geq 7.0 \mathrm{mmol} / \mathrm{L})$ or $2 \mathrm{~h}$ plasma glucose $\geq 200 \mathrm{mg} / \mathrm{dL}$ ( $\geq 11.1 \mathrm{mmol} / \mathrm{L})$ (11). Patients without previously known diabetes but with $\mathrm{HbA} 1 \mathrm{c} \geq 6.5 \%$ on admission were classified as having newly detected DM.

\section{Clinical data collection}

All data was prospectively collected and entered a central database. Measurements of glucose metabolism were made in all patients at hospital admission. According to the definition used in previous studies $(6,12)$, acute hyperglycemia was defined as a blood glucose level at admission $>198 \mathrm{mg} / \mathrm{dL}$ ( $>11 \mathrm{mmol} / \mathrm{L})$. The average glucose levels were estimated by the following formula: [(28.7x HbA1c \%)-46.7] (13). Stress hyperglycemia was defined by the SHR, calculated as acute glycemia divided by estimated average glucose. The estimated glomerular filtration rate was calculated using the modified Diet in Renal Disease equation (14).

\section{Angiographic analysis}

Patients were administered $300 \mathrm{mg}$ aspirin, $180 \mathrm{mg}$ ticagrelor, or $600 \mathrm{mg}$ clopidogrel, and $100 \mathrm{IU} / \mathrm{kg}$ heparin before the interventional procedure. Invasive coronary angiography and PCI were performed according to standard of care on the basis of local practice. The decision for thrombus aspiration in the infarct-related artery during 
the procedure was at the discretion of the operators. The angiographic analysis separately evaluated by 2 experienced interventional cardiologists who were blinded to the blood glucose levels and clinical data. Intracoronary thrombus was angiographically identified by the thrombolysis in myocardial infarction (TIMI) thrombus grade and scored in 5 grades as previous studies reported (15). G0, no thrombus present; G1, possible thrombus present, with angiographic characteristics suggestive of thrombus but not diagnostic of thrombus (i.e., reduced contrast density, haziness, irregular lesion contour or a smooth convex meniscus at the site of total occlusion); G2, definite thrombus present, with greatest dimensions $\leq 0.5$ the vessel diameter; G3, definite thrombus present, with greatest linear dimension $>0.5$ but $<2$ vessel diameters; G4, definite thrombus present, with the largest dimension $\geq 2$ vessel diameters; G5, total occlusion, the size of thrombus cannot be assessed. In patients presenting with an occluded IRA (G5), thrombus was reclassified after flow achievement with either guidewire crossing or a small (diameter $1.5 \mathrm{~mm}$ ) deflated balloon passage or dilation. After reclassification of the G5 group, patients with reclassified G4 or G5 were defined as large thrombus burden (LTB), while G0 to G3 were defined as small thrombus burden (STB). TIMI flow grade and percent diameter stenosis were routinely evaluated at baseline and after the PCI procedure. The number of diseased vessels were counted as the number of coronary arteries with $\geq 50 \%$ stenosis in the three main branches without considering left main artery disease.

\section{Statistical analysis}

Categorical variables are presented as frequencies and proportions and compared between groups using Pearson chi-square or Fisher exact test. Continuous variables are presented as mean $\pm \mathrm{SD}$ or medians (interquartile range, IQR) and compared between groups using Student $t$ tests or Mann-Whitney $U$ test. The diagnostic value of SHR for predicting LTB was analyzed using the receiveroperating characteristic (ROC) curve, with a best cut-off value, sensitivity, specificity, areas under curve (AUC), 95\% confidence intervals $(\mathrm{CI})$, and $\mathrm{p}$ value. As an exploratory analysis, the predictive value of SHR and acute glycemia was analyzed using the ROC curve and compared as recommended by DeLong et al. (16). Patients were then classified by best SHR cut-off value that maximized the sensitivity and specificity for LTB. Univariable logistic regression analysis was performed to determine the odds ratio and $95 \%$ CI for LTB. Multivariable logistical regression analysis was used to adjusted for variables that at univariable analysis had a $\mathrm{P}$ value $\leq 0.15$. A 2 -tailed $\mathrm{P}<0.05$ was considered statistically significant. The statistical analyses were performed using SPSS version 22.0 (SPSS Inc., Chicago, IL, USA).

\section{Results}

\section{Baseline characteristics}

From January, 2017 to December, 2019, a total of 853 patients with STEMI who underwent emergency invasive coronary angiography at Tongji Hospital, Tongji University, Shanghai were prospectively enrolled in this cohort study. A total of 227 STEMI patients with DM met the inclusion and exclusion criteria were included in the present study (Figure 1). Patients' baseline characteristics of the entire population stratified by thrombus burden are presented in Table 1. According to the reclassified TIMI thrombus grades of the entire study population, $77(33.9 \%)$ patients were categorized as LTB group, whereas 150 (66.1\%) patients presented with STB. The mean age was 64.1 years, and $80.6 \%$ of the patients were male. Overall median estimated average glucose level was $172 \mathrm{mg} / \mathrm{dL}$ (146-209 mg/dL). $33.9 \%$ (77) of the entire patients were classified as having newly detected DM and 9.7\% (22) taken insulin treatment. Sex, body mass index, prevalence of smoking, hypertension, dyslipidemia, and the history of the myocardial infarction and PCI were not different between the LTB and STB groups. A comparison of the baseline angiographic and procedural characteristics of the two groups are shown in Table 2. There was a lower rate of initial TIMI flow grade 3 $(\mathrm{P}<0.001)$ and a higher rate of aspiration thrombectomy in the LTB group $(\mathrm{P}<0.001)$, while no difference was observed of rate of TIMI flow grade 3 after procedure. Infarct-related vessels with LTB were more frequently encountered in the right coronary artery (RCA).

\section{Association between SHR and intracoronary thrombus burden}

The levels of acute glycemia [199 (147-263) versus 229 (176-279) $\mathrm{mg} / \mathrm{dL}, \mathrm{P}=0.023]$ and SHR [1.11 (0.96-1.32) versus $1.31(1.13-1.48), \mathrm{P}<0.001]$ were significantly higher in the patients with LTB than in the STB (Figure 2). Figure 3 shows the ROC analysis to compare the predictive value of SHR and acute glycemia for LTB. The SHR 


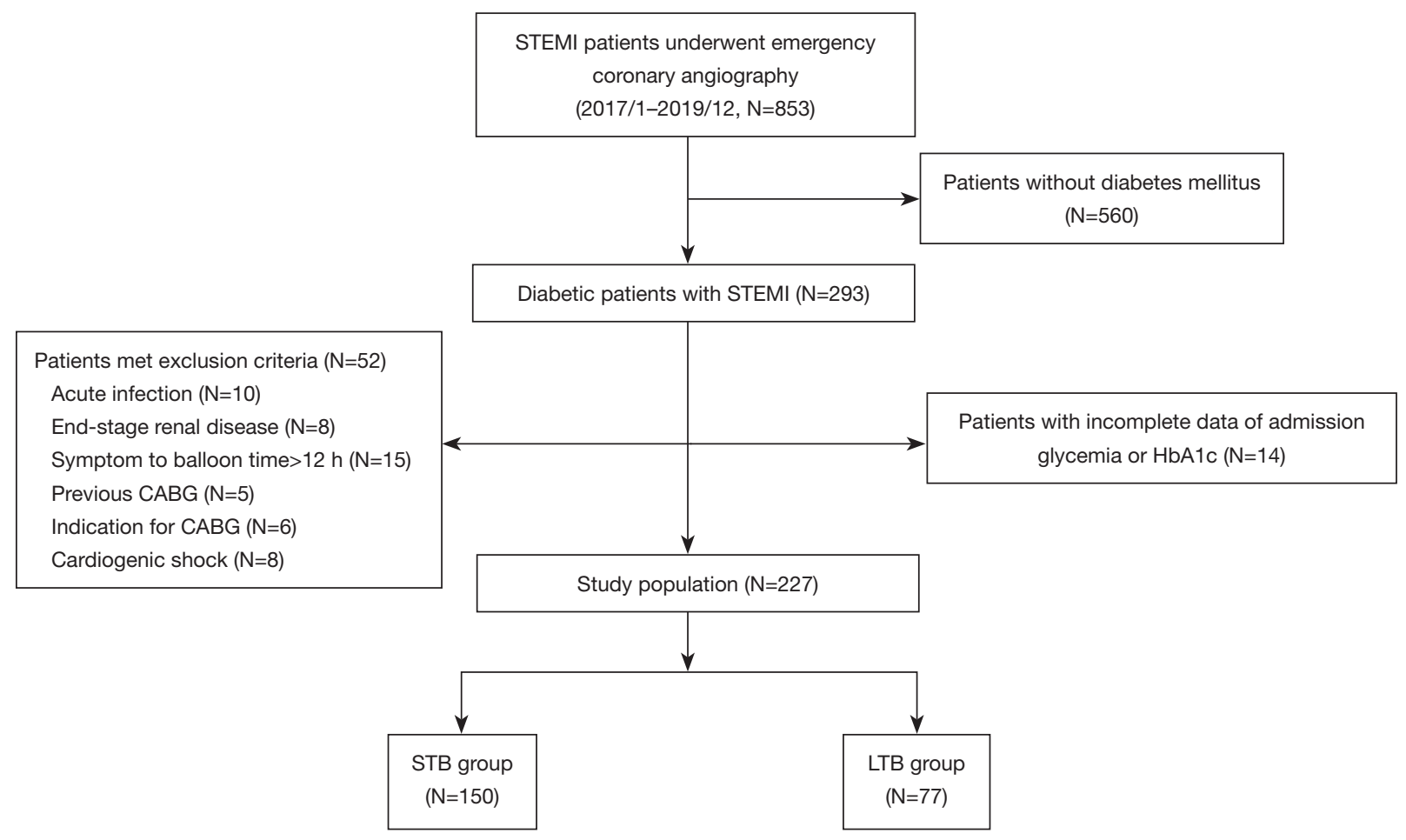

Figure 1 Study flow chart. STEMI, ST-segment elevation myocardial infarction; CABG, coronary artery bypass graft; HbA1c, glycosylated hemoglobin; STB, small thrombus burden; LTB, large thrombus burden.

(AUC: 0.669; 95\% CI: 0.604-0.730; $\mathrm{P}<0.001)$ showed greater accuracy than acute glycemia (AUC: $0.592 ; 95 \%$ CI: $0.525-0.657 ; \mathrm{P}=0.017$ ), the difference of discrimination was significant as assessed by DeLong test (difference: 0.077; 95\% CI: $0.026-0.128 ; \mathrm{P}=0.003)$. The best cut-off value of SHR for predicting LTB was 1.19 and the corresponding sensitivity and specificity were $71.4 \%$ and $64.7 \%$, respectively. The frequency of LTB in the entire patients stratified according to acute glycemia and SHR is shown in Figure 4A. The incidence of LTB increased with the increasing tertiles of SHR levels as follows: $15.6 \%$ in the tertile $1(<1.06), 35.1 \%$ in the tertile $2(1.06-1.13), 49.4 \%$ in the tertile $3(>1.13 ; \mathrm{P}<0.001)$, while no difference was observed in the three acute glycemia tertiles $(24.7 \%$ versus $39.0 \%$ versus $36.4 \%$; $\mathrm{P}=0.127$ ).

\section{Logistic regression analysis}

After stratification into 2 groups according to the cutoff value of SHR, there were $108(47.6 \%)$ patients with a high SHR $(\geq 1.19)$ and $119(52.4 \%)$ patients with a low SHR $(<1.19)$. The rate of LTB was significantly higher in patients with a high SHR than those with a low SHR (50.9\% versus 18.5\%; OR: 4.575; 95\% CI: $2.518-8.313$; $\mathrm{P}<0.001$ ), while there was no significant difference was observed in patients with or without acute hyperglycemia $39.4 \%$ versus 27.0\%; OR: 1.756; 95\% CI: 0.996-3.096; $\mathrm{P}=0.051$ ) (Figure $4 B$ ). Univariable logistic analysis revealed that SHR was significantly associated with the presence of LTB (unadjusted OR: 4.575 ; 95\% CI: $2.518-8.313$; $\mathrm{P}<0.001$ ). Two multivariable models further demonstrated that the SHR remained as an independent predictor of LTB (model 1 OR: 5.962 ; $95 \%$ CI: $2.677-13.275$; $\mathrm{P}<0.001$, model 2 OR: 4.857; 95\% CI: 2.304-10.236; $\mathrm{P}<0.001$ ) (Table 3).

\section{Discussion}

The major findings of the present study are the following: first, in diabetic patients with STEMI undergoing primary PCI, almost $1 / 3$ of them presented with LTB in infarctrelated artery as evaluated by TIMI thrombus grade based on angiographic characteristics. Besides, the SHR levels were significantly higher in patients with LTB than in those with STB. Second, compared with admission glycemia, ROC analysis indicated that SHR may be a better index for distinguishing LTB from STB in diabetic patients with 
Table 1 Baseline clinical characteristics of the study groups

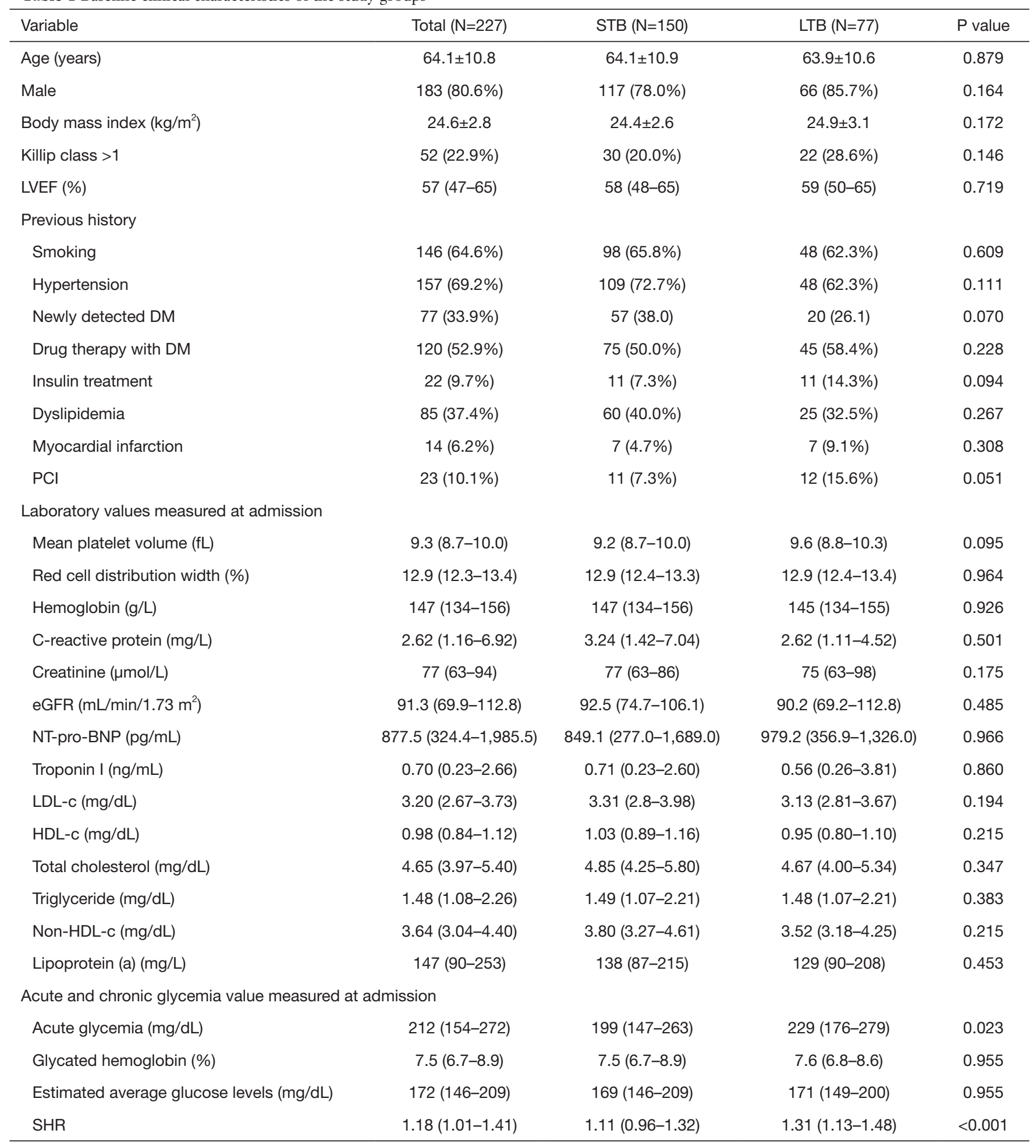

Continuous data are presented as means \pm SD or medians (interquartile ranges), and categorical variables are presented as counts (\%). LVEF, left ventricular ejection fraction; DM, diabetes mellitus; PCI, percutaneous coronary intervention; eGFR, estimated glomerular filtration rate; NT-pro-BNP, N-terminal pro-B-Type natriuretic peptide; LDL-c, low-density lipoprotein cholesterol; HDL-c, high-density lipoprotein cholesterol; SHR, stress hyperglycemia ratio. 
Table 2 Initial periprocedural and angiographic characteristics of the study groups

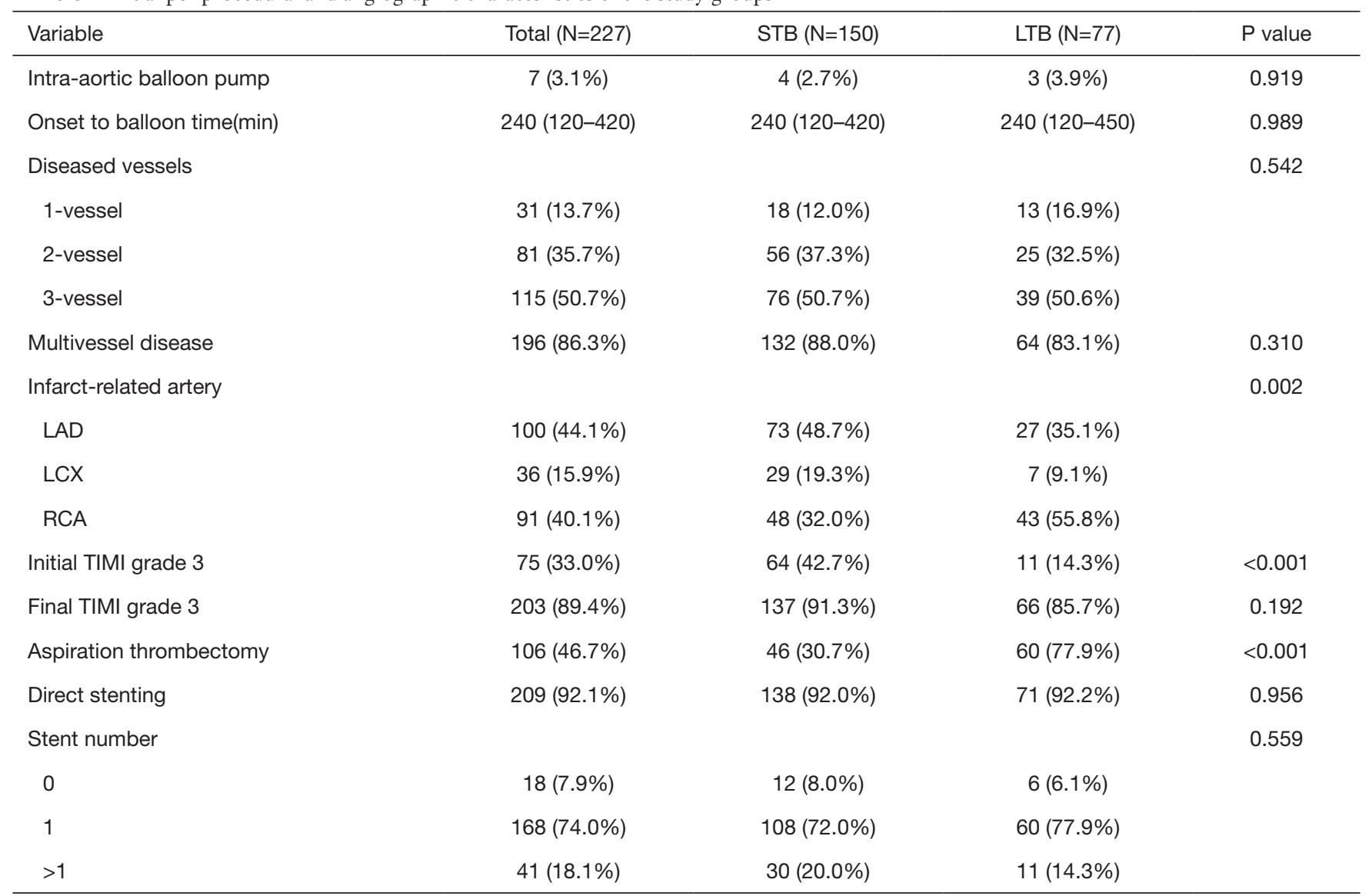

Values are expressed as percentages or median (interquartile range). LAD, left anterior descending coronary artery; LCX, left circumflex; $\mathrm{RCA}$, right coronary artery; TIMI, thrombolysis in myocardial infarction.

A

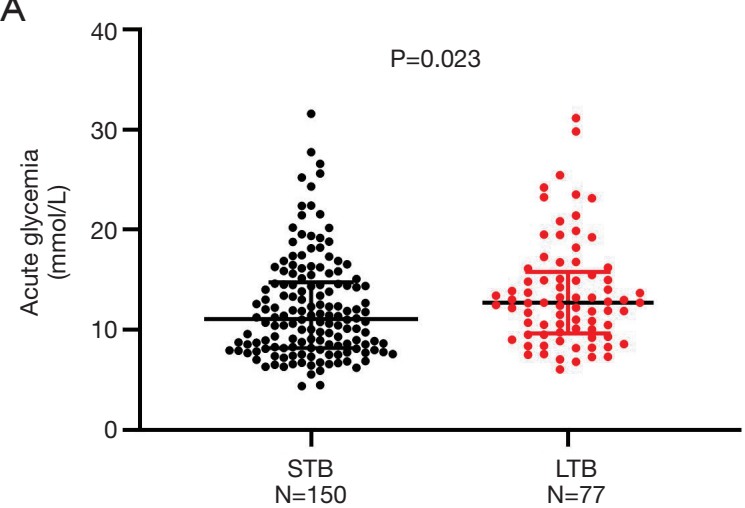

B

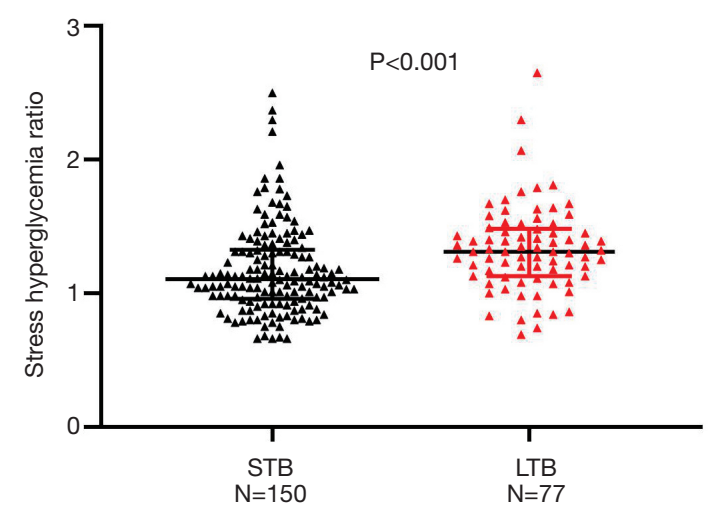

Figure 2 Comparison of acute glycemia (A) and SHR (B) levels between small and large thrombus burden groups. STB, small thrombus burden; LTB, large thrombus burden. 


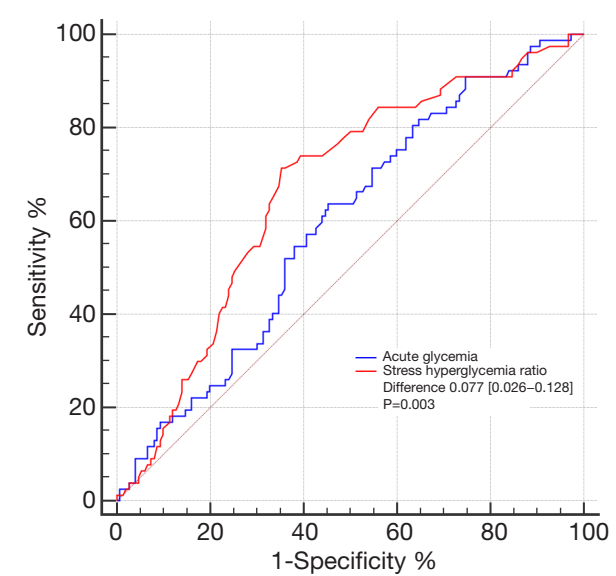

Figure 3 Receiver-operating characteristic curves of acute glycemia and stress hyperglycemia ratio for predicting large thrombus burden.

A

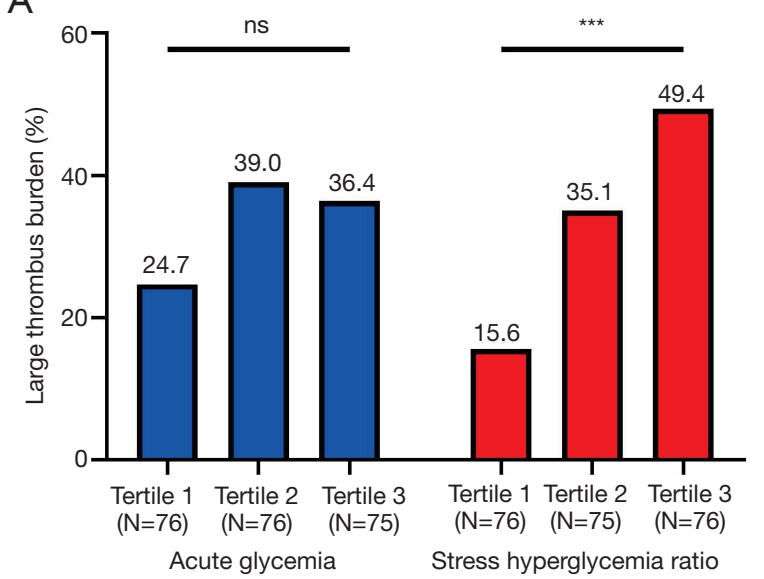

B

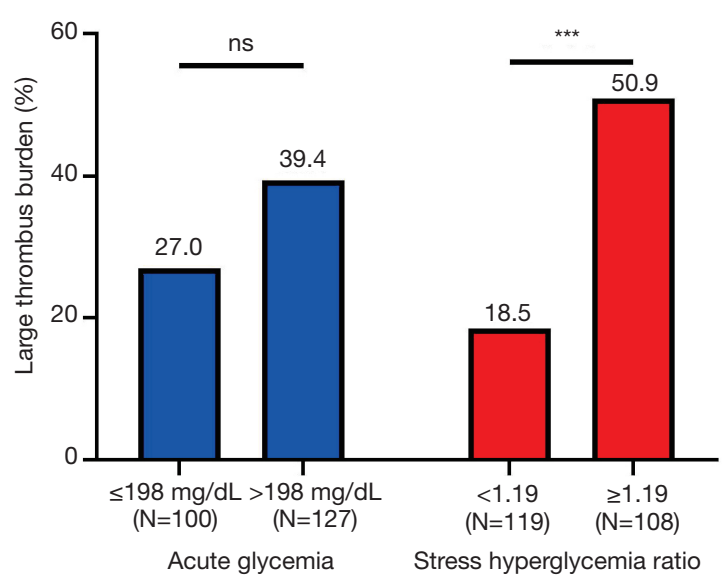

Figure 4 Large thrombus burden incidence stratified according to acute glycemia and stress hyperglycemia ratio. ns, no significant; ***, $\mathrm{P}<0.001$.

STEMI. Third, the high SHR $(\geq 1.19)$ was found to be an independent predictor of LTB following adjustment for baseline clinical confounders.

Rupture or erosion of the vulnerable coronary plaque triggers platelet aggregation and activation of coagulation, resulting subsequent formation of thrombus that leads to AMI (17). The goals of reperfusion strategy, i.e., primary PCI, for patients with acute STEMI are restoring epicardial blood flow and achieving microvascular reperfusion as early as possible, thus maximum salvage of myocardium. However, during primary PCI in patients admitted with STEMI, the presence of intracoronary thrombus burden has been independently associated with impaired myocardial reperfusion, distal embolization and stent thrombosis $(1,3,18)$. LTB is present in a sizeable proportion of patients with STEMI and associated with the increase risk of adverse cardiac events as reported in previous studies $(1,2)$. Therefore, the early determination of predictors for intracoronary thrombus burden is particularly important and may lead us to take appropriate treatment for decreasing thrombus grade before and during procedure.

As reported in several previous studies, increased total bilirubin level, intrinsic platelet reactivity and the absence of pre-infarction angina were found to be independently associated with large angiographic intracoronary thrombus burden (19-21). In a subanalysis of the ACUITY (Acute Catheterization and Urgent Intervention Triage strategY) trial, Goto et al. (18) enrolled 3,267 non-ST- 
Table 3 Univariable and multivariable logistic regression analyses for large thrombus burden

\begin{tabular}{|c|c|c|c|c|}
\hline Variable & \multicolumn{2}{|c|}{ Univariable } & \multicolumn{2}{|c|}{ Multivariable } \\
\hline Hypertension & $0.623(0.347-1.117)$ & 0.112 & 0.709 (0.371-1.358) & 0.300 \\
\hline Newly detected DM & $0.572(0.312-1.050)$ & 0.072 & $0.835(0.414-1.686)$ & 0.616 \\
\hline Insulin treatment & $2.106(0.869-5.106)$ & 0.099 & $1.454(0.526-4.017)$ & 0.470 \\
\hline Killip class >1 & $1.600(0.847-3.022)$ & 0.148 & $1.471(0.725-2.985)$ & 0.285 \\
\hline Acute hyperglycemia* & $1.756(0.996-3.096)$ & 0.052 & $0.580(0.261-1.287)$ & 0.180 \\
\hline Acute hyperglycemia ${ }^{\#}$ & $2.000(1.137-3.517)$ & 0.016 & $0.855(0.405-1.805)$ & 0.681 \\
\hline$S H R \geq 1.19^{*}$ & 4.575 (2.518-8.313) & $<0.001$ & $5.962(2.677-13.275)$ & $<0.001$ \\
\hline
\end{tabular}

Multivariable logistic regression analyses adjusted for variables that at univariable analysis had a $\mathrm{P}$ value $\leq 0.15$ including hypertension, newly detected DM, insulin treatment, previous PCI, Killip class and acute hyperglycemia. *Model 1, acute hyperglycemia was defined as a blood glucose level at admission $>198 \mathrm{mg} / \mathrm{dL}$. "Model 2, acute hyperglycemia defined as the cut-off value $\geq 206 \mathrm{mg} / \mathrm{dL}$. DM, diabetes mellitus; $\mathrm{PCl}$, percutaneous coronary intervention; SHR, stress hyperglycemia ratio.

segment elevation myocardial infarction and found that the independent predictors of pre-procedural thrombus formation included baseline reference diameter, Jeopardy Score, current smoking and right coronary artery disease. In line with this result, the present study revealed that culprit vessels with LTB were more frequently encountered in the RCA in diabetic patients with STEMI. In a very recent study, Sigirci et al. (9) suggested that stress hyperglycemia, defined as admission blood glucose level, is an independent predictive factor of LTB in nondiabetic patients with STEMI. However, the underlying impact of stress hyperglycemia on intracoronary thrombus burden in STEMI patients with DM is still unclear.

Numerous prior studies have established that hyperglycemia on admission is frequently observed in patients with acute coronary syndrome, particularly in the early phase of AMI $(4,5)$. In clinical practice, there is no consensus about the definition of stress hyperglycemia for patients with AMI. Acute hyperglycemia was typically defined as a blood glucose level at admission $>198 \mathrm{mg} / \mathrm{dL}$ as the previous studies reported $(6,12)$. However, in patients with prevalent diabetes, the admission glycemia cannot able to discriminate the acute hyperglycemia from the chronic elevation of glucose levels. Therefore, the SHR was proposed by Roberts et al. (8) as a novel index to evaluate the relative elevation of glycemia and several studies have verified the short-term and long-term prognostic value of the SHR in patients with AMI $(6,7)$. To our best knowledge, the present study is the first to address the relevance of stress hyperglycemia, evaluated by SHR, and thrombus burden in diabetic patients with STEMI.

In our study population with DM and STEMI, we identified a cutoff value $(\geq 1.19)$ for the SHR that is able to discriminate patients at high risk of large intracoronary thrombus burden. Recently, Marenzi et al. (6) analyzed the relative hyperglycemia (defined in their study as acute-tochronic glycemic ratio) in 1553 AMI patients. They found that this ratio is closely associated with in-hospital adverse outcomes, including in-hospital mortality, nonfatal acute pulmonary edema, and cardiogenic shock. In the entire study population, the best cut-point of acute-to-chronic glycemic ratio for in-hospital outcomes was 1.3. Differently from our study, they included all AMI patients and only $27 \%$ with DM. Moreover, the primary endpoint of their study was the combination of in-hospital clinical outcomes. In another very recent study, Zhou et al. (22) found in AMI patients without diabetes, acute hyperglycemia defined as SHR $>1.22$ had a better predictive value for culprit lesion characteristics such as plaque rupture, lipid-rich plaque and thin-cap fibroatheroma using optical coherence tomography. Thus, the thresholds may be different to define acute hyperglycemia depending on the study population and endpoints. As currently no uniform definition of SHR in the setting of AMI, particularly in patients with diabetes. 
The SHR was calculated by combining admission glucose level and average chronic glycemic value estimated from HbA1c, we cannot exclude that the index does not fully reflect acute glycemic changes. Also, the impact on SHR of daily glycemic fluctuations, glucose-lowering treatments, coronary atherosclerotic burden and other factors should be taken into account as a possible bias. For example, in our study, there was a lower rate of initial TIMI flow grade 3 in the LTB group which may potentially influence the level of SHR. Therefore, further studies are needed to validate our findings.

As previous study reported, stress hyperglycemia was associated with no-reflow phenomenon and impaired microvascular function in the setting of AMI, resulting in an increased infarct size and worse functional recovery (23). Furthermore, the no-reflow phenomenon and microvascular obstruction more frequently occurred in STEMI patients with a large thrombus burden after primary PCI. However, the clinical relevance of stress hyperglycemia and intracoronary thrombus has not been well understood. Some studies demonstrated that hyperglycemia leads to a prothrombotic state, induces vascular alterations and inflammatory response, and results in atherothrombosis ultimately $(24,25)$. In an observational study, D'Onofrio et al. (25) analyzed coronary thrombi of 273 hyperglycemic and 273 normal glycemic patients with STEMI undergoing thrombus aspiration during primary PCI. They found the coronary thrombi obtained from hyperglycemic patients showed higher size and increased levels of microRNA-33, inflammatory and coagulative markers compared to normal glycemic patients. Moreover, they suggested that the activation of microRNA-33/sirtuin 1 pathways, by increasing inflammatory and coagulative processes, might be the underlying mechanism for thrombus formation in hyperglycemic patients after STEMI.

Patients with LTB have been thought to benefit most from the strategies aimed at decreasing thrombus grade before stent deployment such as thrombus aspiration. However, the largest trial, TOTAL (ThrOmbecTomy with PCI vs. PCI ALone in patients with STEMI), demonstrated that routine thrombus aspiration does not improve clinical outcomes (26). Moreover, a pre-specified subgroup analysis of TOTAL trial suggested no benefit of routine thrombus aspiration was observed at 1 year in patients with LTB (TIMI thrombus grade $\geq 3$ ). Nevertheless, the authors outlined that LTB is an important and independent predictor of adverse outcome in STEMI (2). Recently, Sardu et al. (27) performed an observational study assessed the thrombus aspiration in 959 hyperglycemic patients during STEMI, they found a benefit of thrombus aspiration for hyperglycemic, but not normoglycemic population. This emphasizes the influence of stress hyperglycemia on coronary thrombus development during STEMI.

In line with the previous clinical studies, our observations suggested that acute glycemic changes have a better predictive value on adverse complications and events compared with admission glucose levels in the setting of AMI $(6,7,9,28)$. Therefore, it is essential to determine the true acute glycemic rise accurately by combing evaluation of acute and chronic glycemic levels, especially in diabetic patients with an elevation of admission glucose level. Moreover, assessment of acute and chronic glycemia in diabetic patients may help physicians more early discern those at high risk for LTB and take appropriate treatment for decreasing thrombus grade and controlling glycemia tightly.

\section{Study limitations}

In this topic, some limitations should be taken into consideration. First, as an observational study at a single center, the sample size was limited and a cause-effect relationship between plasm glucose and intracoronary thrombus burden cannot be determined. Second, in our analysis, the assessment of intracoronary thrombus burden relies on angiography which may be affected by many factors. More reliable methods should be considered for thrombus burden assessment in future and prospective trials, such as optical coherence tomography. Third, despite major clinical confounders were adjusted in the multivariable model, there were possibly residual confounding factors not measured in our study that may affect the thrombus burden. Finally, because of rigorous inclusion and exclusion criteria, a relatively small number of study population enrolled in our study could theoretically result in a selection bias. Conclusions drawn from our findings cannot be extrapolated to these excluded patients. Besides, the impact of stress hyperglycemia and thrombus burden on clinical outcomes was not investigated in the study owing to the small sample size, future studies should make efforts to clarify this causal association.

\section{Conclusions}

To be best of our knowledge, we demonstrated for the first time that a high SHR value $(\geq 1.19)$ was independently associated with large thrombus burden and has a better 
predictive value than glycemia at admission in diabetic patients with STEMI undergoing primary PCI. The combined evaluation of acute and chronic glycemic levels is helpful to improve risk stratification and management for diabetic patients with STEMI. In the meantime, our observations suggest that stress hyperglycemia may contribute to the intracoronary thrombus formation in the setting of STEMI. Further studies are required to elucidate the exact mechanism of stress hyperglycemia in large coronary thrombus.

\section{Acknowledgments}

Funding: This study was supported by the National Natural Science Foundation of China (Grant No. 81670403), Grant of Shanghai Science and Technology Committee (NO. 18411950300, 19XD1403300 and 1941196320), and the Shanghai Municipal Health Commission (NO. 2019LJ10).

\section{Footnote}

Reporting Checklist: The authors have completed the STROBE reporting checklist. Available at http://dx.doi. org/10.21037/jtd-20-2111

Data Sharing Statement: Available at http://dx.doi. org/10.21037/jtd-20-2111

Conflicts of Interest: All authors have completed the ICMJE uniform disclosure form (available at http://dx.doi. org/10.21037/jtd-20-2111). The authors have no conflicts of interest to declare.

Ethical Statement: The authors are accountable for all aspects of the work in ensuring that questions related to the accuracy or integrity of any part of the work are appropriately investigated and resolved. This study was conducted in accordance with the Declaration of Helsinki (as revised in 2013). The study was approved by ethics board of Tongji Hospital of Tongji University (No. KYSB-2014-054) and informed consent was taken from all the patients.

Open Access Statement: This is an Open Access article distributed in accordance with the Creative Commons Attribution-NonCommercial-NoDerivs 4.0 International License (CC BY-NC-ND 4.0), which permits the noncommercial replication and distribution of the article with the strict proviso that no changes or edits are made and the original work is properly cited (including links to both the formal publication through the relevant DOI and the license). See: https://creativecommons.org/licenses/by-nc-nd/4.0/.

\section{References}

1. Sianos G, Papafaklis MI, Daemen J, et al. Angiographic stent thrombosis after routine use of drug-eluting stents in ST-segment elevation myocardial infarction: the importance of thrombus burden. J Am Coll Cardiol 2007;50:573-83.

2. Jolly SS, Cairns JA, Lavi S, et al. Thrombus Aspiration in Patients With High Thrombus Burden in the TOTAL Trial. J Am Coll Cardiol 2018;72:1589-96.

3. Napodano M, Dariol G, Al Mamary AH, et al. Thrombus Burden and Myocardial Damage During Primary Percutaneous Coronary Intervention. Am J Cardiol 2014;113:1449-56.

4. Planer D, Witzenbichler B, Guagliumi G, et al. Impact of hyperglycemia in patients with ST-segment elevation myocardial infarction undergoing percutaneous coronary intervention: the HORIZONS-AMI trial. Int J Cardiol 2013;167:2572-9.

5. Kojima T, Hikoso S, Nakatani D, et al. Impact of Hyperglycemia on Long-Term Outcome in Patients With ST-Segment Elevation Myocardial Infarction. Am J Cardiol 2020;125:851-9.

6. Marenzi G, Cosentino N, Milazzo V, et al. Prognostic Value of the Acute-to-Chronic Glycemic Ratio at Admission in Acute Myocardial Infarction: A Prospective Study. Diabetes Care 2018;41:847-53.

7. Gao S, Liu Q, Ding X, et al. Predictive Value of the Acuteto-Chronic Glycemic Ratio for In-Hospital Outcomes in Patients With ST-Segment Elevation Myocardial Infarction Undergoing Percutaneous Coronary Intervention. Angiology 2020;71:38-47.

8. Roberts GW, Quinn SJ, Valentine N, et al. Relative Hyperglycemia, a Marker of Critical Illness: Introducing the Stress Hyperglycemia Ratio. J Clin Endocrinol Metab 2015;100:4490-7.

9. Sigirci S, Yildiz SS, Keskin K, et al. The predictive value of stress hyperglycemia on thrombus burden in nondiabetic patients with ST-segment elevation myocardial infarction. Blood Coagul Fibrinolysis 2019;30:270-6.

10. Thygesen K, Alpert JS, Jaffe AS, et al. Fourth universal definition of myocardial infarction (2018). Eur Heart J 2019;40:237-69.

11. American Diabetes Association. 2. Classification and Diagnosis of Diabetes. Diabetes Care 2019;42:S13-S28. 
12. Wahab NN, Cowden EA, Pearce NJ, et al. Is blood glucose an independent predictor of mortality in acute myocardial infarction in the thrombolytic era? J Am Coll Cardiol 2002;40:1748-54.

13. Nathan DM, Kuenen J, Borg R, et al. Translating the A1C assay into estimated average glucose values. Diabetes Care 2008;31:1473-8.

14. Levey AS, Bosch JP, Lewis JB, et al. A more accurate method to estimate glomerular filtration rate from serum creatinine: a new prediction equation. Modification of Diet in Renal Disease Study Group. Ann Intern Med 1999;130:461-70.

15. Gibson CM, de Lemos JA, Murphy SA, et al. Combination therapy with abciximab reduces angiographically evident thrombus in acute myocardial infarction: a TIMI 14 substudy. Circulation 2001;103:2550-4.

16. DeLong ER, DeLong DM, Clarke-Pearson DL. Comparing the areas under two or more correlated receiver operating characteristic curves: a nonparametric approach. Biometrics 1988;44:837-45.

17. Libby P, Pasterkamp G, Crea F, et al. Reassessing the Mechanisms of Acute Coronary Syndromes. Circ Res 2019;124:150-60.

18. Goto K, Lansky AJ, Nikolsky E, et al. Prognostic significance of coronary thrombus in patients undergoing percutaneous coronary intervention for acute coronary syndromes: a subanalysis of the ACUITY (Acute Catheterization and Urgent Intervention Triage strategY) trial. JACC Cardiovasc Interv 2011;4:769-77.

19. Hamur H, Duman H, Bakirci EM, et al. Bilirubin Levels and Thrombus Burden in Patients With ST-Segment Elevation Myocardial Infarction. Angiology 2016;67:565-70.

20. Alexopoulos D, Xanthopoulou I, Tsigkas G, et al. Intrinsic platelet reactivity and thrombus burden in patients with ST-elevation myocardial infarction. Thromb Res

Cite this article as: Chu J, Tang J, Lai Y, Gao Y, Ye Z, Guan C, Ding K, Yao Y, Chen F, Liu X. Association of stress hyperglycemia ratio with intracoronary thrombus burden in diabetic patients with ST-segment elevation myocardial infarction. J Thorac Dis 2020;12(11):6598-6608. doi: 10.21037/jtd20-2111
2013;131:333-7.

21. Ahmed TA, Sorgdrager BJ, Cannegieter SC, et al. Preinfarction angina predicts thrombus burden in patients admitted for ST-segment elevation myocardial infarction. EuroIntervention 2012;7:1396-405.

22. Zhou J, Sheng Z, Liu C, et al. Association between Admission Hyperglycemia and Culprit Lesion Characteristics in Nondiabetic Patients with Acute Myocardial Infarction: An Intravascular Optical Coherence Tomography Study. J Diabetes Res 2020;2020:1763567.

23. Iwakura K, Ito H, Ikushima M, Kawano S, et al. Association between hyperglycemia and the no-reflow phenomenon in patients with acute myocardial infarction. J Am Coll Cardiol 2003;41:1-7.

24. Worthley MI, Holmes AS, Willoughby SR, et al. The deleterious effects of hyperglycemia on platelet function in diabetic patients with acute coronary syndromes mediation by superoxide production, resolution with intensive insulin administration. J Am Coll Cardiol 2007;49:304-10.

25. D'Onofrio N, Sardu C, Paolisso P, et al. MicroRNA-33 and SIRT1 influence the coronary thrombus burden in hyperglycemic STEMI patients. J Cell Physiol 2020;235:1438-52.

26. Jolly SS, Cairns JA, Yusuf S, et al. Randomized trial of primary PCI with or without routine manual thrombectomy. N Engl J Med 2015;372:1389-98.

27. Sardu C, Barbieri M, Balestrieri ML, et al. Thrombus aspiration in hyperglycemic ST-elevation myocardial infarction (STEMI) patients: clinical outcomes at 1-year follow-up. Cardiovasc Diabetol 2018;17:152.

28. Marenzi G, Cosentino N, Milazzo V, et al. Acute Kidney Injury in Diabetic Patients With Acute Myocardial Infarction: Role of Acute and Chronic Glycemia. J Am Heart Assoc 2018;7:e008122. 